\title{
A cross-species drug discovery pipeline to identify and validate new treatments for osteosarcoma
}

3

Jason A. Somarelli\#*, Gabrielle Rupprecht*, Erdem Altunel, Etienne M. Flamant, Sneha Rao, Dharshan Sivaraj, Alexander L. Lazarides, Sarah M. Hoskinson, Maya U. Sheth, Serene Cheng, So Young Kim, Kathryn E. Ware, Anika Agarwal, Mark M. Cullen, Laura E. Selmic, Jeffrey I.

7 Everitt, Shannon J. McCall, Cindy Eward, William C. Eward ${ }^{+}$, and S. David Hsu ${ }^{+}$

$9 *$ these authors contributed equally

10 these authors contributed equally

11

\#Address correspondence to: jason.somarelli@duke.edu

14 Key words: comparative oncology, patient-derived xenografts, precision medicine, CRM1,

proteasome, sarcoma

16

17 Conflict of interest statement: The authors declare no conflicts of interest. 


\section{Abstract}

31 Purpose: Osteosarcoma is a rare but aggressive bone cancer that occurs primarily in children.

32 Like other rare cancers, treatment advances for osteosarcoma have stagnated, with little

33 improvement in survival for the past several decades. Developing new treatments has been

34 hampered by extensive genomic heterogeneity and limited access to patient samples to study the

35 biology of this complex disease. Experimental design: To overcome these barriers, we

36 combined the power of comparative oncology with patient-derived models of cancer and high-

37 throughput chemical screens in a cross-species drug discovery pipeline. Results: Coupling in

38 vitro high-throughput drug screens on low-passage and established cell lines with in vivo

39 validation in patient-derived xenografts we identify the proteasome and CRM1 nuclear export

40 pathways as therapeutic sensitivities in osteosarcoma, with dual inhibition of these pathways

41 inducing synergistic cytotoxicity. Conclusions: These collective efforts provide an experimental

42 framework and set of new tools for osteosarcoma and other rare cancers to identify and study

43 new therapeutic vulnerabilities. 


\section{Introduction}

54 Osteosarcoma, the most common primary bone cancer, exemplifies the progress that

55 needs to be made in the approach to discovering new therapies. As the third most common

56 cancer of childhood, osteosarcoma is disproportionately lethal, and patients with advanced or

57 metastatic disease have limited treatment options [1]. Due to the low incidence of osteosarcoma

58 and the extensive genetic heterogeneity [2], finding common genetic drivers and common

59 pathways of relevance remains difficult, and the exact etiology remains unknown. Because of

60 these features, progress in identifying new therapies has stagnated, and decades of research have

61 brought almost no improvement in patient survival rates [3, 4]. Even for those patients who

62 survive, both their life expectancy and quality of life are negatively impacted by the current

63 treatment regimen [5]. For all of these reasons, there is a persistently unmet need to develop new

64 therapies for this deadly disease.

65 The story of osteosarcoma is the story of many cancers, especially uncommon ones:

66 Alternative therapeutic approaches are urgently needed, but the path forward is not clear. How

67 can we identify, design, and test new molecular therapies in a disease that is both rare and

68 genetically diverse? Mouse models are a critical tool, but additional translational steps are

69 needed. To complete these additional steps, we are able to look to dogs with osteosarcoma.

70 While there are approximately 800 cases of human osteosarcoma diagnosed each year, there are

71 at least 30,000 cases of canine osteosarcoma diagnosed each year $[6,7]$. Treatments in canine

72 and human osteosarcoma patients are identical, and studies have revealed remarkable genomic

73 conservation between canine and human osteosarcoma, with shared molecular alteration of

74 known cancer pathways and shared amplifications of known oncogenes [8-11]. Canine patients

75 with spontaneous disease - in contrast to genetically-engineered mouse models - offer a high 
76 incidence of spontaneous tumors that are comparable to humans biologically and genetically [7,

77 12], share environmental factors with humans, have an intact immune system, and possess

78 similar clinical presentation including progression, resistance, recurrence, and metastasis. Most

79 importantly, canine osteosarcoma patients have a shorter course of disease than human

80 osteosarcoma patients, which means therapeutic discoveries could be made more quickly with a

81 platform that integrates canine osteosarcoma into our current disease models (reviewed in [13]).

82 Naturally-occurring osteosarcoma in dogs offers an unparalleled opportunity to understand the

83 genomics of the disease, to learn about disease progression, and to trial new investigational drugs

84 that would otherwise take too long to accrue in human studies.

85 Patient-derived models of cancer, including low-passage cell lines [14], patient-derived

86 organoids [15, 16], and patient-derived xenografts (PDXs) [17], are increasingly being used as

87 "standard" preclinical models to identify sensitivities to new candidate therapeutics across

88 cancers. Patient-derived xenografts are also being used to predict drug response [18] and identify

89 novel drug combinations [19]. Organoid models are also now being developed to test response to

90 immunotherapies, as the organoids for several cancer types have been shown to contain

91 infiltrating lymphocytes [20,21]. Combinations of these patient-derived models are currently

92 being explored to develop precision medicine strategies for cancer care [22]. However,

93 translating new discoveries in real time remains a challenge in human patients, in whom disease

94 progression can be slow and whose overall picture can be complicated by a variety of treatments,

95 both for the cancer and for comorbid conditions.

96 Here we combine the advantages of a comparative oncology approach (e.g. larger

97 numbers of patients, fewer confounding treatment variables, more rapid disease progression)

98 with patient-derived models to develop and refine a cross-species drug discovery pipeline. The 
99 pipeline uses patient samples from either pet dogs or humans to generate patient-matched, low-

100 passage cell lines, and PDXs. Cell lines are used to perform high-throughput chemical screens,

101 and top hits are validated in vivo using matched PDX models (Figure 1A). Using this approach,

102 we identified the proteasome and CRM1 nuclear export pathways as therapeutic vulnerabilities

103 for osteosarcoma. Using in vitro and in vivo validations, we show that inhibition of both the

104 proteasome and CRM1 pathways acts synergistically to inhibit osteosarcoma growth. Together,

105 these results demonstrate the utility of our cross-species drug discovery pipeline to identify new

106 targets and strategies to treat osteosarcoma and other rare cancers.

107

108 Results

109 Development of a cross-species drug discovery pipeline.

110 Osteosarcomas and other rare cancers suffer from a lack of access to patient-derived models for

111 study. We reasoned that increased access to a larger patient population with nearly-identical

112 biology could have significant benefits in identifying actionable pathways in osteosarcoma. To

113 this end, we developed a cross-species pipeline that leverages the increased canine patient

114 population and the extensive biological similarities between humans and pet dogs with naturally-

115 occurring sarcomas. The pipeline uses patient tumor tissue from dogs or humans to create

116 patient-derived xenografts (PDXs) that are grown and passaged in immunocompromised mice

117 (Figure 1A). These PDXs are used to create matched, low-passage, patient-derived cell lines.

118 The cell lines are applied to high-throughput screens to identify candidate therapies, and top

119 candidates are validated in vivo using patient-matched PDXs (Figure 1A) to identify therapeutic

120 vulnerabilities that are shared across species. To date, we have created a total of 9 human

121 (Figure 1B) and 20 canine sarcoma PDXs (Figure 1C). 
A

124

125

126

127

128
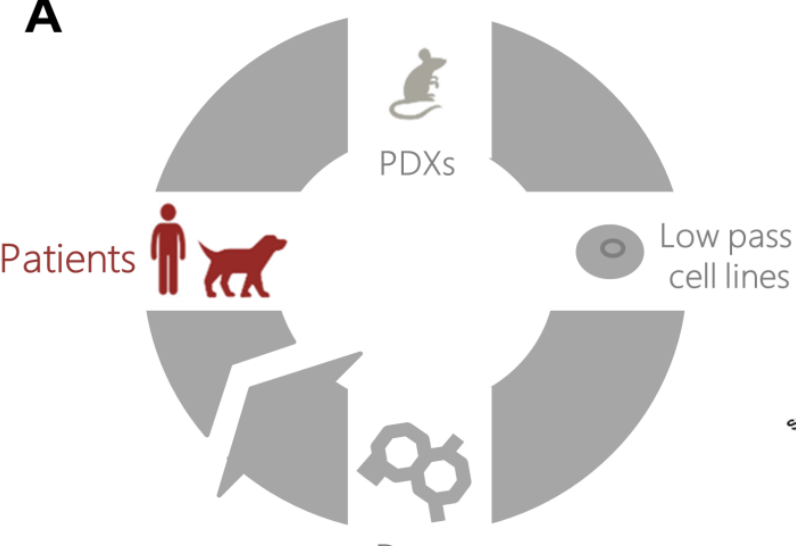

B
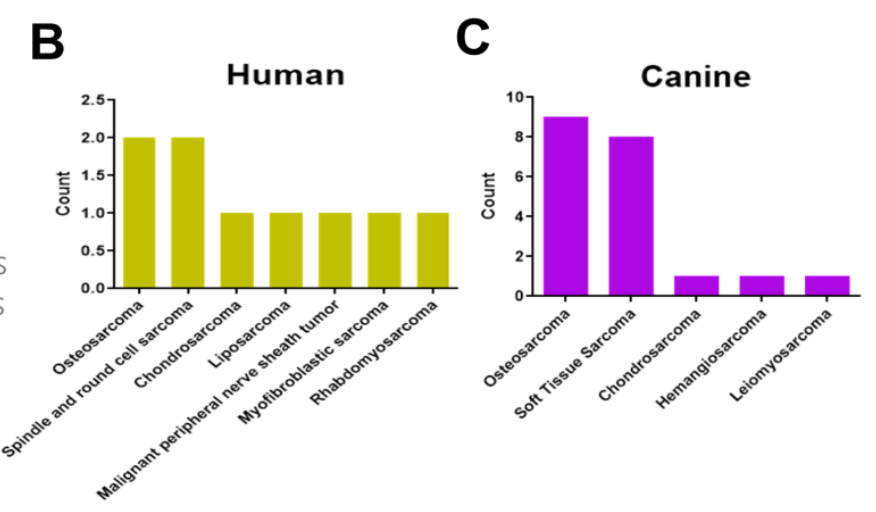

Figure 1. A cross-species personalized medicine pipeline using patient-derived models of cancer. A. The pipeline uses tumor samples from human and canine patients to establish matched patient-derived xenografts and low-passage cell lines. The cell lines are used in high-throughput drug screens, and results from the screen are validated in matched patient-derived xenografts. B. A summary of human (top) and $\mathbf{C}$. dog (bottom) samples obtained and number of patient-derived xenografts created.

The cross-species pipeline identified CRM1 and proteasome inhibition as novel treatments for

osteosarcoma. We applied our pipeline to reveal novel therapeutic vulnerabilities at both the individual patient level and, more importantly, shared across osteosarcomas. To do this, we created matched cell lines from both dog (D418) and human (17-3X) PDXs. Both PDXs were confirmed as osteosarcoma by histopathology (Figure 2A, B). Using the PDX tissue, we created clonally-purified cell lines. The 17-3X human osteosarcoma cell line exhibits a more spindleshaped morphology while the D418 dog osteosarcoma cell line displays a rounded, cobblestonelike morphology (Figure 2A, B, right panels). Clonally-derived cell lines were confirmed to be free of detectable mouse fibroblasts using species-specific PCR assays designed to detect human, dog, and mouse DNA (Figure 2C, D). The 17-3X cell line has an estimated doubling time of approximately 40 hours (Figure 2E) while the D418 line grows more rapidly, with an estimated doubling time of approximately 21 hours (Figure 2F). The 17-3X and D418 low-passage cell 

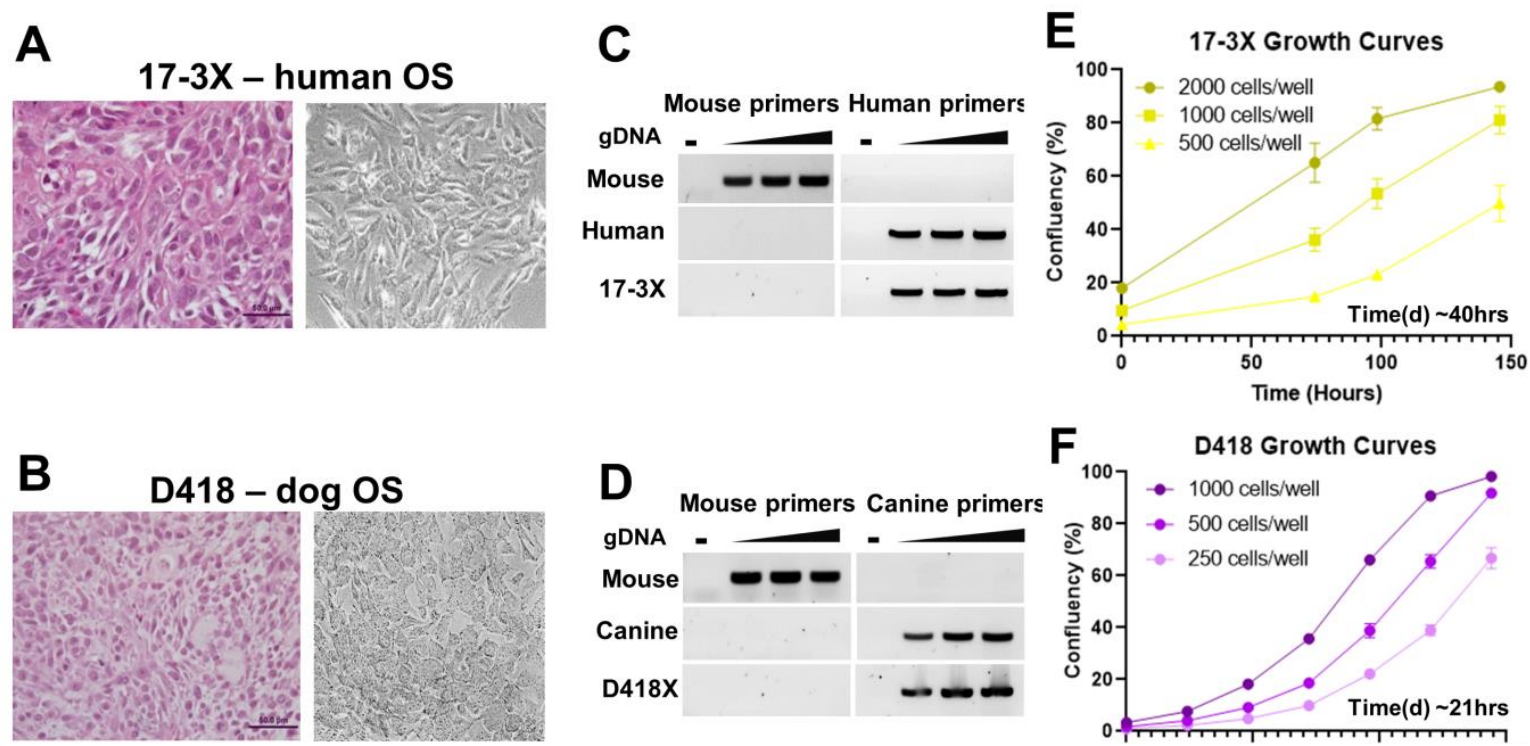

Figure 2. Cross-species analysis of drug activity reveals remarkable similarity in response. A.

Establishment of matched patient-derived xenografts and cell lines from human (17-3X) and $\mathbf{B}$. dog (D418) osteosarcomas. C. and D. Species-specific PCRs are used to verify the cell lines are purified cancer cell lines devoid of mouse fibroblast contamination. E. The estimated doubling times for the 17-3X and D418 cell lines are approximately 40 and F. 21 hours, respectively.

146 lines were combined with a panel of seven additional established osteosarcoma cell lines from

147 both humans and dogs to perform high-throughput screens using 119 FDA-approved small

148 molecule oncology drugs. These screens revealed several trends: 1) cell line-specific variation in

149 responses were observed across the panel, with some cell lines more broadly resistant to drugs in

150 the screen, such as U2OS and D17, and other lines more sensitive, such as 17-3X and Abrams

151 (Figure 3A); 2) hierarchical clustering of the nine cell lines using the drug responses for each

152 cell line revealed species-specific clustering of the cell lines, with distinct human and dog clades

153 formed based on response of each cell line to the entire panel of drugs (Figure 3A); 3) while cell

154 lines clustered by species, there was overall consistency in the average percent killing across all

155 cell lines, particularly among the top hits (Figure 3B). Together, these results suggest that

156 although both individual and species-specific responses exist across osteosarcomas, there are

157 consistent responses to the most effective agents. Importantly, among the top hits were standard-

158 of-care agents such as anthracyclines (e.g., doxorubicin, daunorubicin, idarubicin, epirubicin) 
and methotrexate, which consistently killed all cell lines, while others showed wider variation

160 (e.g., etoposide) and limited efficacy (e.g., platinum-based chemotherapy) (Figure 3C). These

161 analyses indicate that this screening approach is capable of identifying relevant therapies for

162 osteosarcoma.

164 individual cell line level revealed heterogeneous responses across the panel of nine cell lines

165 (Figure 3D). Most drugs, such as belinostat (an HDAC inhibitor) or ponatinib (a multi-tyrosine

166 kinase inhibitor), were efficacious across more than one of the nine cell lines (Figure 3D). These

167 analyses reveal that substantial heterogeneity in drug response exists across osteosarcomas.
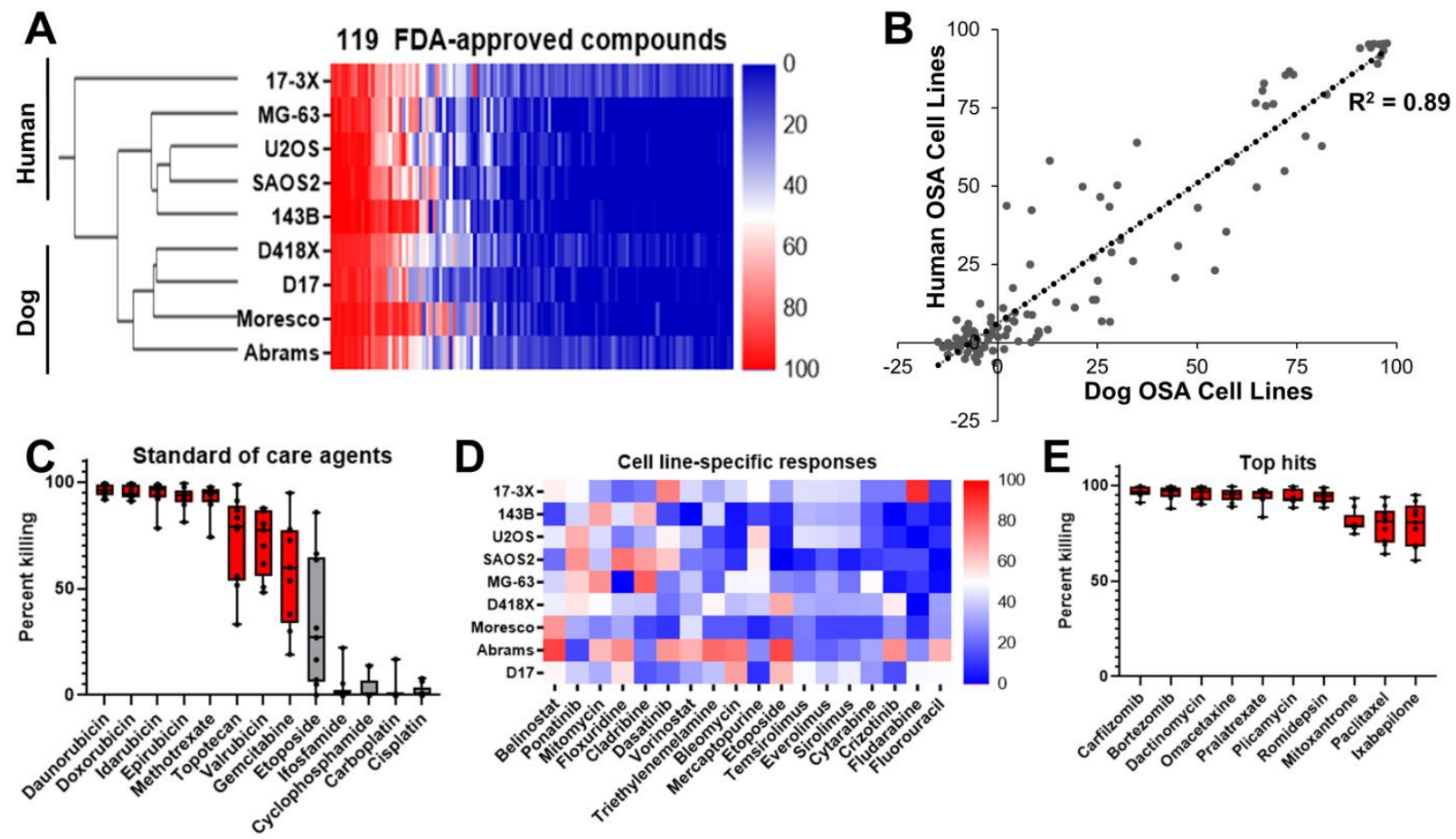

Figure 3. Cross-species analysis of osteosarcoma drug response reveals sensitivity to proteasome inhibition. A. A high-throughput screen of 119 oncology compounds across nine osteosarcoma cell lines revealed species-specific clustering by drug response. B. Although both individual and species-specific responses exist across osteosarcomas, there is a strong correlation between dog and human cell lines $\left(\mathrm{R}^{2}=\right.$ 0.89). C. Standard-of-care agents, such as anthracyclines and methotrexate are among the top hits. D. Cellline specific responses vary widely to targeted agents and other chemotherapeutics. E. Proteasome inhibitors carfilzomib and bortezomib demonstrate efficacy across all nine cell lines. 
with efficacy across the entire panel of cell lines. Interestingly, the two most efficacious

170 inhibitors, with an average of $>95 \%$ killing for both inhibitors across all nine lines, were

171 compounds that target the proteasome pathway (Figure 3E). Together, our results indicate that

172 both human and canine osteosarcomas display heterogeneity in drug response across cell lines,

173 with convergence on the proteasome pathway as a novel target to treat osteosarcoma.

To better understand the landscape of therapeutic vulnerabilities in osteosarcoma, we next performed high-throughput chemical screens in D418 and 17-3X patient-derived lines using

177 enabling both protein- and pathway-level interrogation of chemical sensitivities. D418 and 17-

$1783 \mathrm{X}$ cells displayed similar sensitivity profiles, with just $11.9 \%$ and $8.7 \%$ of compounds inducing

$179 \geq 50 \%$ killing in D418 and 17-3X, respectively (Figure 4A, B). Consistent with the results from

180 the 119 compound screens, responses of D418 and 17-3X to all 2,100 compounds were

181 correlated $\left(\mathrm{R}^{2}=0.54 ; \mathrm{p}<0.0001\right)($ Figure 4C). Also consistent with the previous screens, each

182 cell line displayed sensitivity to a subset of agents (Figure 4D). For example, D418 displayed

183 sensitivity to multiple MEK and FAK inhibitors while 17-3X was uniquely sensitive to Chk

184 inhibitors (Figure 4D). In addition to the unique sensitivities, both cell lines showed common

185 sensitivity to standard-of-care anthracyclines and a number of novel agents (Figure 4E). These

186 agents included the zinc pyrithione, the active ingredient in dandruff shampoo, the pan-selective

187 Jumonji histone demethylase inhibitor, JIB-04, an NF-kB inhibitor (WS3), and two CDK

188 inhibitors (alvocidib and SNS-032) (Figure 4E). Given that almost all small molecule inhibitors

189 have multiple targets, we focused on targets for which at least three drugs showed $>50 \%$ killing.

190 We reasoned that filtering by drug targets with multiple hits in the screen would identify the

191 most high-confidence drug targets for downstream validation. From these analyses, we identified 
192 the CRM1 nuclear export and proteasome pathways as the top candidate targets (Figure 4F). A

193 total of 3 of 4 CRM1 inhibitors and 9 of 11 proteasome inhibitors showed $>50 \%$ killing in both

194 D418 and 17-3X cell lines (Figure 4G, H). Consistent with these analyses, both the CRM1

195 inhibitor, verdinexor, and the proteasome inhibitor, bortezomib, showed dose-dependent

196 inhibition of 143B and 17-3X human osteosarcomas (Figure 4I, J) and D418 and D17 canine

197 osteosarcomas (Figure 4K, L), pinpointing the CRM1 and proteasome pathways as lead

198 candidates for in vivo validation.
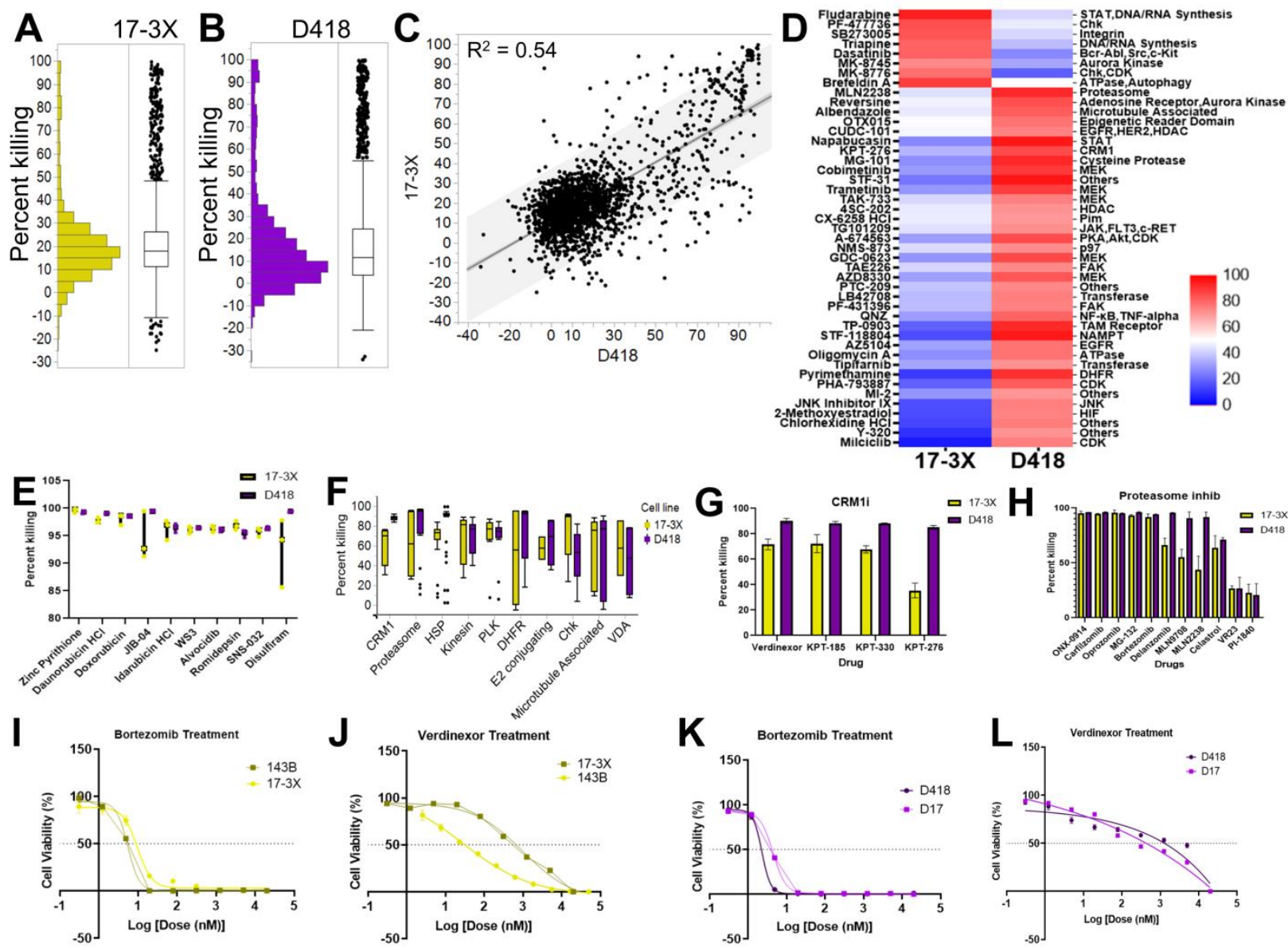

Figure 4. Interrogating the therapeutic landscape of osteosarcoma pinpoints the proteasome and nuclear export pathways as promising therapeutic targets. A. Chemical screens were performed using 2,100 compounds in 17-3X and B. D418 low-passage cell lines. C. Drug response was correlated across species $\left(\mathrm{R}^{2}=0.54\right)$. D. Cell line-specific sensitivities for 17-3X and D418 cell lines. E. Top drugs, and $\mathbf{F}$. top pathways for both cell lines. G. Cell line-specific response to each of the CRM1 inhibitors and $\mathbf{H}$. proteasome inhibitors. 
200 In vivo validation of proteasome and CRM1 pathway inhibitors to treat osteosarcoma

201 Our in vitro small molecule screens pinpointed the proteasome and CRM1 nuclear export

202 pathways as two promising therapeutic vulnerabilities for osteosarcoma. Consistent with this,

203 CRM1 protein is highly expressed (Supplementary Figure 1A) and localized within the nucleus

204 of osteosarcoma cells (Supplementary Figure 1B). Moreover, elevated CRM1 expression is

205 prognostic for poorer metastasis-free and overall survival in human osteosarcoma

206 (Supplementary Figure 1C, D). We validated the therapeutic efficacy of proteasome and

207 CRM1 inhibition in D418 PDXs and showed that both CRM1 inhibition and proteasome

208 inhibition significantly reduced tumor growth (Figure 5A). CRM1 inhibition also significantly

209 reduced 17-3X PDX tumor growth, while the proteasome inhibitor, bortezomib, had no effect as

210 a single agent (Figure 5B). Mouse weights remained unchanged during the course of the

211 treatment (Supplementary Figure E, F). Based on the positive results for CRM1 inhibitors in

212 two PDXs, we further verified the efficacy of CRM1 inhibition in two additional PDXs, D071

213 and D075 (Figure 5C, D).

214

215 Combined proteasome and CRM1 pathway inhibition act synergistically to prevent osteosarcoma

216 growth.

217 Our cross-species pipeline pinpointed both CRM1 and proteasome pathways as promising

218 single-agent therapies to treat osteosarcoma. However, although CRM1 inhibition was effective

219 across all four of the PDXs tested, the proteasome inhibitor, bortezomib, was only capable of

220 inhibiting growth in one of the two treated PDXs when used as a single agent. Interestingly,

221 these pathways are being targeted with combination therapy for synergistic benefit in several 
223 Based on these studies and our results, we hypothesized that combined CRM1 and proteasome

224 blockade would have synergistic benefit for osteosarcoma. Consistent with this hypothesis, the

225 combination of CRM1 and proteasome inhibition induced synergistic cell death for both D418

226 and 17-3X cells (Figure 5E, F), suggesting that combined inhibition of the proteasome and

227 CRM1 pathways may represent a rational strategy to treat osteosarcoma.

A

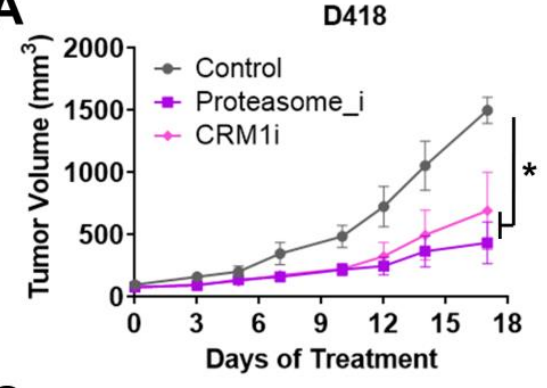

C

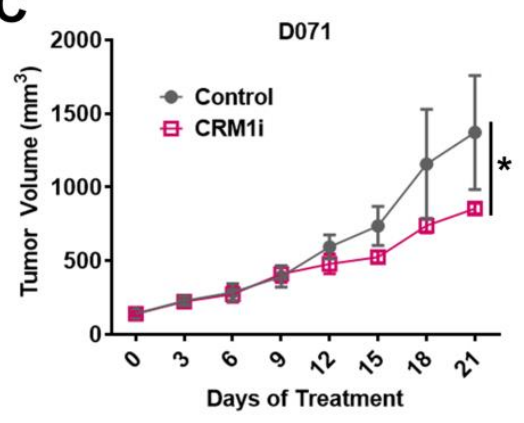

E



B

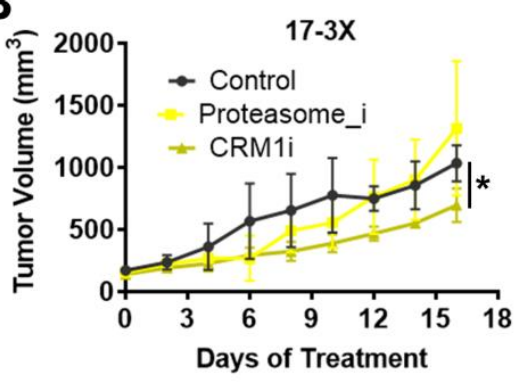

D

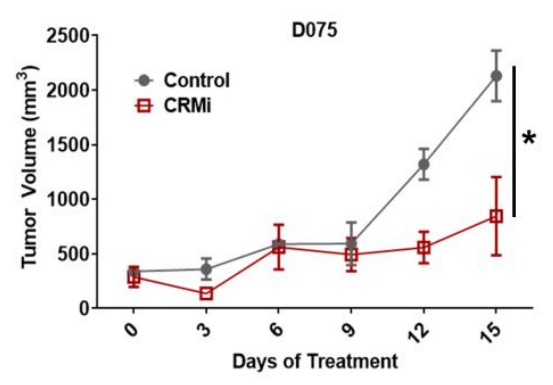

$17-3 x$

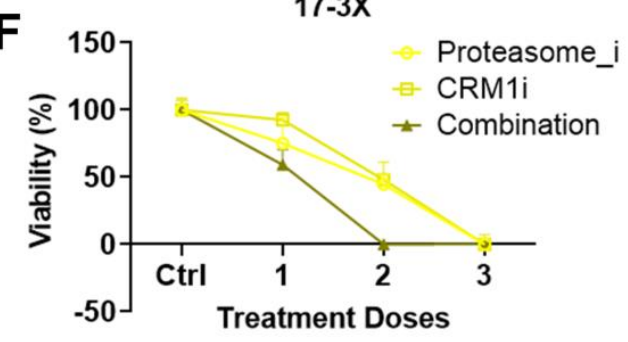

Figure 5. Proteasome and CRM1 nuclear export pathway inhibition reduces osteosarcoma tumor growth and induces synergistic killing of osteosarcomas. A. CRM1 inhibition (verdinexor), but not proteasome inhibition (bortezomib) significantly reduced tumor growth in 17-3X. B. Both CRM1 and proteasome inhibition significantly reduced D418 tumor growth. C. and D. Combined CRM1 and proteasome inhibition led to synergistic inhibition of 17-3X and D. D418 cell growth. 


\section{Discussion}

In the present study, we sought to combine the utility of comparative oncology with patient-derived models of cancer and high-throughput small molecule screens to create a cross-

231 species, personalized medicine pipeline. We applied this pipeline to osteosarcoma, a painful

232 bone cancer that occurs predominantly in adolescents and for which almost no treatment

233 progress has been made in nearly four decades. Using our pipeline, we identified therapeutic

234 vulnerabilities for osteosarcoma that are patient-specific, common across patients, and common

235 across species. Among the common therapeutic sensitivities identified were the proteasome and

236 CRM1 nuclear export pathways. Both of these pathways have been explored in depth as potential

237 cancer monotherapies for a range of cancer types [26, 27], and both of these therapies are

238 currently approved by the U.S. Food and Drug Administration for the treatment of multiple 239 myeloma [28, 29].

The proteasome is a multi-subunit complex that degrades misfolded, damaged, or unused

241 proteins. The proteasome regulates the turnover of thousands of proteins in the cell [30], and

242 proteasome inhibition creates an imbalance in the levels of misfolded proteins, leading to

243 induction of the unfolded protein response, cellular stress, and apoptosis [31]. The CRM1

244 nuclear export pathway transports proteins through the nuclear envelope to the cytoplasm [32].

245 Like the proteasome, the nuclear export pathway is responsible for regulating thousands of

246 proteins in the cell [33]. Interestingly, the proteasome and CRM1 export pathways are

247 functionally linked. Proteasome inhibition in colorectal cancer cells induces CRM1-dependent

248 nuclear export of ubiquitinated proteins, and inhibition of CRM1 prevents this export, leading to

249 cell cycle arrest and apoptosis [24]. In addition, CRM1 inhibition re-sensitizes chemo-resistant

250 myeloma cells to proteasome inhibition [34]. Inhibiting these inter-dependent pathways 
251 synergistically inhibits multiple cancers [23-25]. Consistent with observations in other cancers,

252 our data support the investigation of proteasome and CRM1 pathway inhibitors for

253 osteosarcoma. In addition, combined proteasome and CRM1 pathway inhibition led to

254 synergistic cytotoxicity in vitro, providing further evidence for the efficacy of dual proteasome

255 and CRM1 pathway inhibition in treating osteosarcoma.

The establishment of a cross-species drug discovery pipeline provides a robust platform

257 to identify and validate potential new therapies and offers several advantages. First, it capitalizes

258 on the expanded canine patient population with spontaneous disease, thereby substantially

259 improving access to patient samples for the development of patient-derived models of cancer.

260 This is particularly useful for studying rare cancers. Indeed, we established more than double the

261 number of PDXs from canines than from humans. Second, combining low passage and

262 established cell lines with high-throughput chemical screens enables interrogation of hundreds to

263 thousands of compounds simultaneously, pinpointing both patient-specific and population-level

264 therapeutic vulnerabilities. Third, the use of chemical screens with complete target and pathway

265 annotation allows for identification of single agent- and target/pathways-level drug sensitivities.

266 Fourth, the use of matched cell lines and PDXs from the same patient provides a robust in vivo

267 system to validate top candidates. While the in vivo studies are a critical validation step in the

268 pipeline, these studies are costly and time consuming. Future iterations of the pipeline that

269 exploit continued improvement in patient-derived models, such as patient-derived organoids [16,

$27035,36]$, are sure to improve the speed and cost-effectiveness of the pipeline and will further

271 enable rapid translation of lead candidates into clinical practice [37]. Fifth, and perhaps most

272 critically, the ability to test these candidates in veterinary clinical trials "closes the loop" of drug 
273 discovery (Figure 1A) and enables testing of novel therapeutic strategies at a fraction of the cost

274 and time necessary for human trials (reviewed in [13]).

275

276 Materials and methods

277 Generation of the patient- derived xenograft models

278 Canine sarcoma tumor tissue samples were collected from University of Illinois at Urbana

279 Collage of Veterinary Medicine (Urbana, IL, USA) and Triangle Veterinary Referral Hospital

280 (Durham, NC, USA) under Institutional Animal Care and Use committee (IACUC)-approved

281 protocols. Human sarcoma tumor tissue samples were collected under a Duke IRB approved

282 protocol (Pro00002435). All patients provided written informed consent to participate in the

283 study. PDX models of the human and canine sarcoma were generated as described previously

284 [37] and all in vivo mouse experiments were performed in accordance with the animal guidelines

285 and with Duke University IACUC approval. To develop PDXs, human and canine tumor tissue

286 samples were washed in phosphate buffered saline (PBS), dissected into small pieces $(<2 \mathrm{~mm})$,

287 and injected into the flanks of 8-10-week-old JAX NOD.CB17-PrkdcSCID-J mice obtained from

288 the Duke University Rodent Genetic and Breeding Core.

291 Patient derived cell line generation, authentication and detection of mouse cell contamination

292 Cell lines were generated from human and canine tumor samples. After washing in phosphate

293 buffered saline (PBS), small pieces $(<2 \mathrm{~mm})$ of tumor tissue were mechanically homogenized.

294 The homogenized tissue was then suspended in cell growth media and cultured in 12 well plates

295 with DMEM + 10\% FBS + 1\% Penicillin/Streptomycin. To isolate tumor cells, growing colonies 
296 of cells were isolated by trypsinization using O rings and cultured in new 12 well plates. This

297 process was repeated until a colony of cells that resembled pure tumor cells in morphology was

298 established.

299 For the cell lines generated from PDX, mouse cell contamination of the PDX cell lines

300 were detected by PCR using human, canine, and mouse specific primers. Because mouse primers

301 easily cross-react with canine and human gDNA, two different mouse primer sets were used. For

302 human cell lines, human reverse (5' TCC AGG TTT ATG GAG GGT TC), human forward (5'

303 TAG ACA TCG TAC TAC ACG ACA CG), mouse reverse (5' CCC AAA GAA TCA GAA

304 CAG ATG C) and mouse forward (5’ ATT ACA GCC GTA CTG CTC CTA T); for canine cell

305 lines, canine reverse (5' GTA AAG GCT GCC TGA GGA TAA G), canine forward (5'GGT

306 CCA GGG AAG ATC AGA AAT G), mouse reverse (AGG TGT CAC CAG GAC AAA TG)

307 and mouse forward (CTG CTT CGA GCC ATA GAA CTA A) primer sets were used.

308 All PDX and patient derived cell lines were authenticated using the Duke University DNA

309 Analysis Facility cell line authentication (CLA) service by analyzing DNA samples from each

310 individual cell line for polymorphic short tandem repeat (STR) markers using the GenePrint 10

311 kit from Promega (Madison, WI, USA).

314 In vitro studies and high-throughput drug screen

315 All human (143B, MG63, SAOS, U2OS, 17-3X) and canine (Abrams, Moresco, D17, D418)

316 osteosarcoma cell lines were cultured in DMEM + 10\% FBS + 1\% Penicillin/Streptomycin. The

317 NIH Approved Oncology set (119 compounds) and Selleck Bioactives collection (2,100

318 compounds) were screened in the Duke Functional Genomics Shared Resource as described 
previously [37, 38]. Briefly, compounds were first stamped in triplicate into 384 well plates for a

320 final concentration of $1 \mu \mathrm{M}$ using an Echo Acoustic Dispenser (Labcyte). Cells and media were

321 then dispensed into plates using a WellMate (Thermo Fisher) at a density of 2,000 cells/well for

322 each cell line. CellTiter-Glo (Promega) viability assays were performed after incubation of cells

323 with compounds for 72 hours and luminescence was read using a Clariostar plate reader (BMG).

324 Top drug targets as identified by the high-throughput drug screens, Bortezomib (PS-341),

325 Verdinexor (KPT-335) and 17-DMAG (Alvespimycin) HCl were purchased from Selleck

326 Chemicals (Houston, TX) and were solubilized in DMSO at $10 \mathrm{mM}$ concentration to use for in

327 vitro IC50 studies.

329 In vivo drug sensitivity validation

330 To validate in vitro drug screen results in vivo, $150 \mu$ homogenized PDX tissue-PBS

331 suspensions at $150 \mathrm{mg} / \mathrm{ml}$ concentration were injected subcutaneously into the right flanks of the

3328 weeks old JAX NOD.CB17- PrkdcSCID-J mice. When the tumor volumes reached $100 \mathrm{~mm}^{3}$,

333 mice were randomized ( $n=5$ mice for each treatment group and $n=5$ for the control group) and

$3341 \mathrm{mg} / \mathrm{kg}$ bortezomib, $25 \mathrm{mg} / \mathrm{kg}$ alvespimycin and $5 \mathrm{mg} / \mathrm{kg}$ verdinexor intraperitoneal injections

335 were initiated twice a week. Tumor volumes were measured three times a week using calipers,

336 and $\frac{\left(\text { Length } x \text { Width }^{2}\right)}{2}$ was used to calculate the tumor size. Mice were sacrificed on day 18 or if

337 the tumor volume reached $1500 \mathrm{~mm}^{3}$.

$339 \quad$ Data analysis and statistics

340 JMP from SAS software (Cary, NC, USA) was used for the high-throughput drug screen data

341 analysis. Analysis of Means was used to identify the top drug candidates from the 119- 
compound drug screen and the 2,100-compound screen. Tumor volumes were recorded in

\section{3}

344

345

GraphPad Prism 6 software (La Jolla, CA, USA). Two-way ANOVA analysis was used to compare the tumor volumes among the control and treatment groups.

\section{Acknowledgments}

This work was supported by the Triangle Center for Evolutionary Medicine (JAS), Hyundai Hope on Wheels (WCE), the Ryan Foundation (WCE), the Consortium for Canine Comparative Oncology (WCE/JAS), and an Orthopedic Research and Education Foundation Resident Research Grant (ALL). The authors acknowledge and thank the BioRepository \& Precision Pathology Center (BRPC), a shared resource of the Duke University School of Medicine and Duke Cancer Institute, for providing access to human biospecimens used under institutional review board oversight in this work. The BRPC receives support from the P30 Cancer Center Support Education Foundation Resident Research Grant (P30 CA014236ALL). The authors acknowledge the Duke Functional Genomics Shared Resource for assistance with compound screens.

\section{Figure legends}

Figure 1. A cross-species personalized medicine pipeline using patient-derived models of cancer. A. The pipeline uses tumor samples from human and canine patients to establish matched patient-derived xenografts and low-passage cell lines. The cell lines are used in highthroughput drug screens, and results from the screen are validated in matched patient-derived xenografts. B. A summary of human (top) and C. dog (bottom) samples obtained and number of patient-derived xenografts created.

Figure 2. Cross-species analysis of drug activity reveals remarkable similarity in response. A. Establishment of matched patient-derived xenografts and cell lines from human (17-3X) and B. $\operatorname{dog}$ (D418) osteosarcomas. C. and D. Species-specific PCRs are used to verify the cell lines are purified cancer cell lines devoid of mouse fibroblast contamination. E. The estimated doubling times for the 17-3X and D418 cell lines are approximately 40 and F. 21 hours, respectively.

\section{Figure 3. Cross-species analysis of osteosarcoma drug response reveals sensitivity to} proteasome inhibition. A. A high-throughput screen of 119 oncology compounds across nine osteosarcoma cell lines revealed species-specific clustering by drug response. B. Although both individual and species-specific responses exist across osteosarcomas, there is a strong correlation between dog and human cell lines $\left(\mathrm{R}^{2}=0.89\right)$. C. Standard-of-care agents, such as anthracyclines and methotrexate are among the top hits. D. Cell-line specific responses vary widely to targeted 
agents and other chemotherapeutics. E. Proteasome inhibitors carfilzomib and bortezomib demonstrate efficacy across all nine cell lines.

Figure 4. Interrogating the therapeutic landscape of osteosarcoma pinpoints the proteasome and nuclear export pathways as promising therapeutic targets. A. Chemical screens were performed using 2,100 compounds in 17-3X and B. D418 low-passage cell lines. C. Drug response was correlated across species $\left(R^{2}=0.54\right)$. D. Cell line-specific sensitivities for 17-3X and D418 cell lines. E. Top drugs, and F. top pathways for both cell lines. G. Cell linespecific response to each of the CRM1 inhibitors and H. proteasome inhibitors.

Figure 5. Proteasome and CRM1 nuclear export pathway inhibition reduces osteosarcoma tumor growth and induces synergistic killing of osteosarcomas. A. CRM1 inhibition (verdinexor), but not proteasome inhibition (bortezomib) significantly reduced tumor growth in 17-3X. B. Both CRM1 and proteasome inhibition significantly reduced D418 tumor growth. C. and D. Combined CRM1 and proteasome inhibition led to synergistic inhibition of 17-3X and D. D418 cell growth.

Supplementary Figure 1. CRM1 upregulation is prognostic for poorer clinical outcomes in osteosarcomas. A. The CRM1 protein is expressed in both human and canine osteosarcoma, and B. Immunofluorescence staining reveals nuclear localization of CRM1 in human and canine osteosarcoma cells. C. Elevated CRM1 mRNA expression is prognostic for poorer metastasisfree and D. overall survival in human osteosarcomas. E. The average weight of mice with D418 PDXs treated with bortezomib or verdinexor remained unchanged during treatment. F. The average weight of mice with 17-3X PDXs treated with bortezomib or verdinexor remained unchanged during treatment. 


\section{References}

427 1. Mirabello, L., R.J. Troisi, and S.A. Savage, International osteosarcoma incidence patterns in children and adolescents, middle ages and elderly persons. Int J Cancer, 2009. 125(1): p. 229-34.

2. $\quad$ Egas-Bejar, D., et al., Theranostic Profiling for Actionable Aberrations in Advanced High Risk Osteosarcoma with Aggressive Biology Reveals High Molecular Diversity: The Human Fingerprint Hypothesis. Oncoscience, 2014. 1(2): p. 167-179.

3. Friebele, J.C., et al., Osteosarcoma: A Meta-Analysis and Review of the Literature. Am J Orthop (Belle Mead NJ), 2015. 44(12): p. 547-53.

4. He, H., J. Ni, and J. Huang, Molecular mechanisms of chemoresistance in osteosarcoma (Review). Oncol Lett, 2014. 7(5): p. 1352-1362.

5. Armenian, S. and S. Bhatia, Predicting and Preventing Anthracycline-Related Cardiotoxicity. Am Soc Clin Oncol Educ Book, 2018. 38: p. 3-12.

6. Rodriguez, C.O., Jr., Using canine osteosarcoma as a model to assess efficacy of novel therapies: can old dogs teach us new tricks? Adv Exp Med Biol, 2014. 804: p. 237-56.

7. Schiffman, J.D. and M. Breen, Comparative oncology: what dogs and other species can teach us about humans with cancer. Philos Trans R Soc Lond B Biol Sci, 2015. 370(1673).

8. Paoloni, M., et al., Canine tumor cross-species genomics uncovers targets linked to osteosarcoma progression. BMC Genomics, 2009. 10: p. 625.

9. Karlsson, E.K., et al., Genome-wide analyses implicate 33 loci in heritable dog osteosarcoma, including regulatory variants near CDKN2A/B. Genome Biol, 2013. 14(12): p. R132.

10. Angstadt, A.Y., et al., Characterization of canine osteosarcoma by array comparative genomic hybridization and RT-qPCR: signatures of genomic imbalance in canine osteosarcoma parallel the human counterpart. Genes Chromosomes Cancer, 2011. 50(11): p. 859-74.

11. Angstadt, A.Y., et al., A genome-wide approach to comparative oncology: highresolution oligonucleotide aCGH of canine and human osteosarcoma pinpoints shared microaberrations. Cancer Genet, 2012. 205(11): p. 572-87.

12. Lindblad-Toh, K., et al., Genome sequence, comparative analysis and haplotype structure of the domestic dog. Nature, 2005. 438(7069): p. 803-19.

13. Somarelli, J.A., et al., Improving Cancer Drug Discovery by Studying Cancer across the Tree of Life. Mol Biol Evol, 2020. 37(1): p. 11-17.

14. van de Wetering, M., et al., Prospective derivation of a living organoid biobank of colorectal cancer patients. Cell, 2015. 161(4): p. 933-45.

15. Barretina, J., et al., The Cancer Cell Line Encyclopedia enables predictive modelling of anticancer drug sensitivity. Nature, 2012. 483(7391): p. 603-7.

16. Vlachogiannis, G., et al., Patient-derived organoids model treatment response of metastatic gastrointestinal cancers. Science, 2018. 359(6378): p. 920-926.

17. Koga, Y. and A. Ochiai, Systematic Review of Patient-Derived Xenograft Models for Preclinical Studies of Anti-Cancer Drugs in Solid Tumors. Cells, 2019. 8(5).

18. Gao, H., et al., High-throughput screening using patient-derived tumor xenografts to predict clinical trial drug response. Nat Med, 2015. 21(11): p. 1318-25.

19. Lu, M., et al., Activation of the mTOR Pathway by Oxaliplatin in the Treatment of Colorectal Cancer Liver Metastasis. PLoS One, 2017. 12(1): p. e0169439. 
20. Scognamiglio, G., et al., Patient-derived organoids as a potential model to predict response to PD-1/PD-L1 checkpoint inhibitors. Br J Cancer, 2019. 121(11): p. 979-982.

21. Neal, J.T., et al., Organoid Modeling of the Tumor Immune Microenvironment. Cell, 2018. 175(7): p. 1972-1988 e16.

22. Pauli, C., et al., Personalized In Vitro and In Vivo Cancer Models to Guide Precision Medicine. Cancer Discov, 2017. 7(5): p. 462-477.

23. Turner, J.G., et al., XPO1 inhibitor combination therapy with bortezomib or carfilzomib induces nuclear localization of IkappaBalpha and overcomes acquired proteasome inhibitor resistance in human multiple myeloma. Oncotarget, 2016. 7(48): p. 7889678909.

24. Wu, T., et al., Nuclear Export of Ubiquitinated Proteins Determines the Sensitivity of Colorectal Cancer to Proteasome Inhibitor. Mol Cancer Ther, 2017. 16(4): p. 717-728.

25. Kashyap, T., et al., Selinexor, a Selective Inhibitor of Nuclear Export (SINE) compound, acts through NF-kappaB deactivation and combines with proteasome inhibitors to

26. Mofers, A., et al., Proteasome-associated deubiquitinases and cancer. Cancer Metastasis Rev, 2017. 36(4): p. 635-653.

27. Sun, Q., et al., Inhibiting cancer cell hallmark features through nuclear export inhibition.

Signal Transduct Target Ther, 2016. 1. p. 16010.

28. XPO1 Inhibitor Approved for Multiple Myeloma. Cancer Discov, 2019. 9(9): p. 11501151.

29. Kane, R.C., et al., United States Food and Drug Administration approval summary: bortezomib for the treatment of progressive multiple myeloma after one prior therapy. Clin Cancer Res, 2006. 12(10): p. 2955-60.

30. Kim, W., et al., Systematic and quantitative assessment of the ubiquitin-modified proteome. Mol Cell, 2011. 44(2): p. 325-40.

31. Obeng, E.A., et al., Proteasome inhibitors induce a terminal unfolded protein response in multiple myeloma cells. Blood, 2006. 107(12): p. 4907-16.

32. Stade, K., et al., Exportin 1 (Crmlp) is an essential nuclear export factor. Cell, 1997.

33. Taylor, J., et al., Altered Nuclear Export Signal Recognition as a Driver of Oncogenesis. Cancer Discov, 2019. 9(10): p. 1452-1467.

34. Chanukuppa, V., et al., XPO1 is a critical player for bortezomib resistance in multiple myeloma: A quantitative proteomic approach. J Proteomics, 2019. 209: p. 103504.

35. Bruun, J., et al., Patient-Derived Organoids from Multiple Colorectal Cancer Liver Metastases Reveal Moderate Intra-patient Pharmacotranscriptomic Heterogeneity. Clin Cancer Res, 2020.

36. Ooft, S.N., et al., Patient-derived organoids can predict response to chemotherapy in metastatic colorectal cancer patients. Sci Transl Med, 2019. 11(513).

37. Rao, S.R., et al., From the Clinic to the Bench and Back Again in One Dog Year: How a Cross-Species Pipeline to Identify New Treatments for Sarcoma Illuminates the Path Forward in Precision Medicine. Front Oncol, 2020. 10: p. 117.

38. $\mathrm{Xu}, \mathrm{S}$., et al., An Integrative Systems Biology and Experimental Approach Identifies Convergence of Epithelial Plasticity, Metabolism, and Autophagy to Promote Chemoresistance. J Clin Med, 2019. 8(2). 
A Patients
B

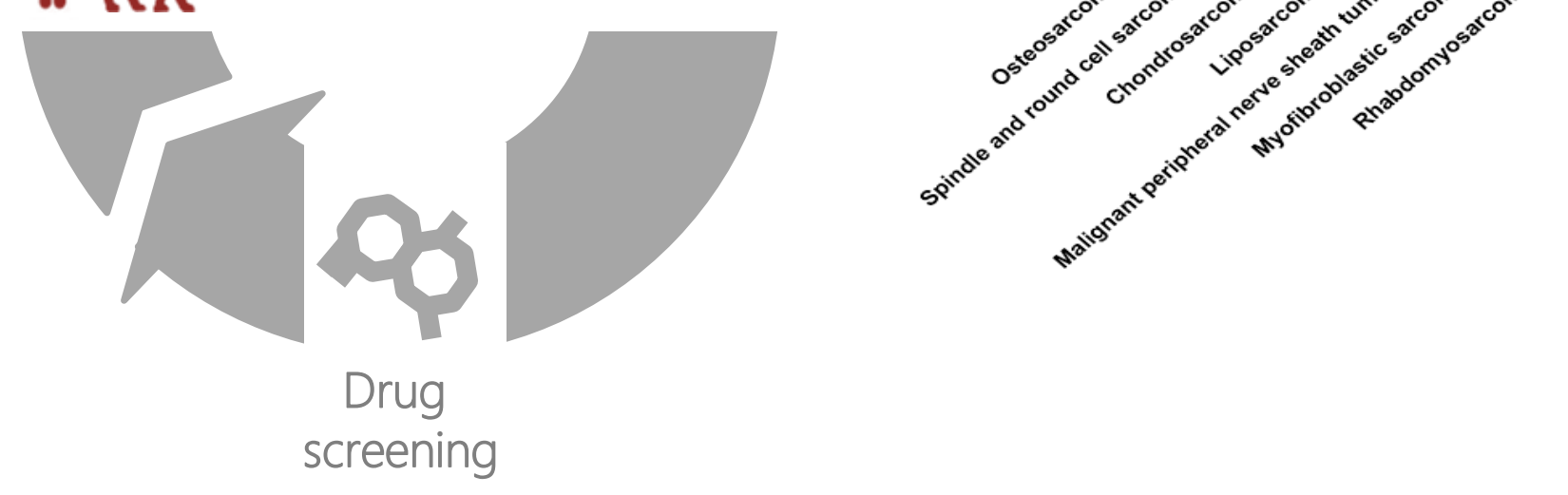

C

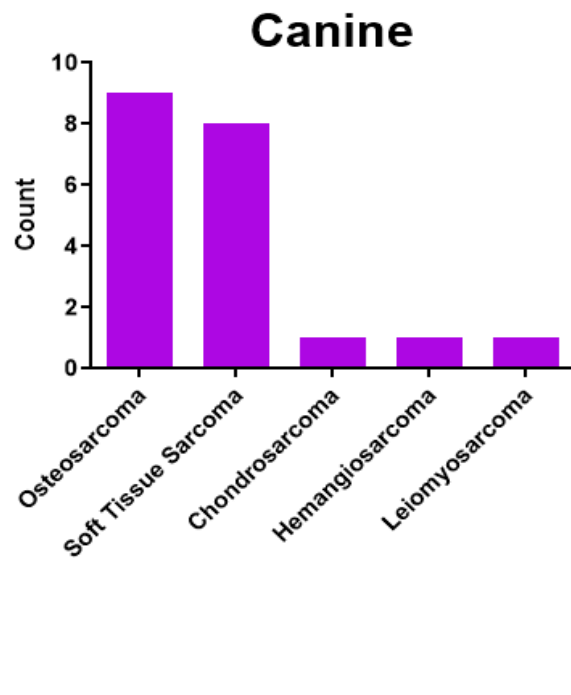




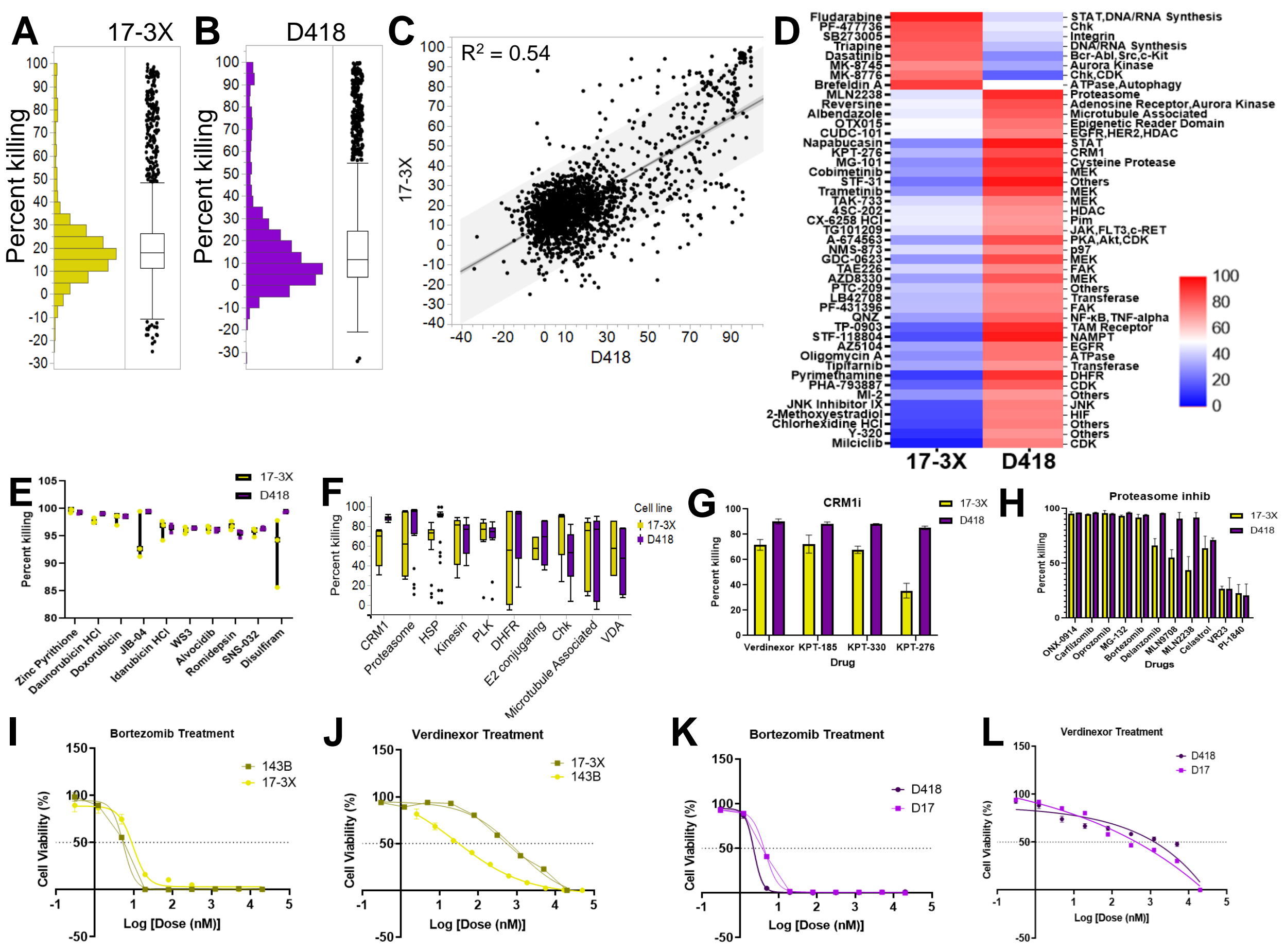


A

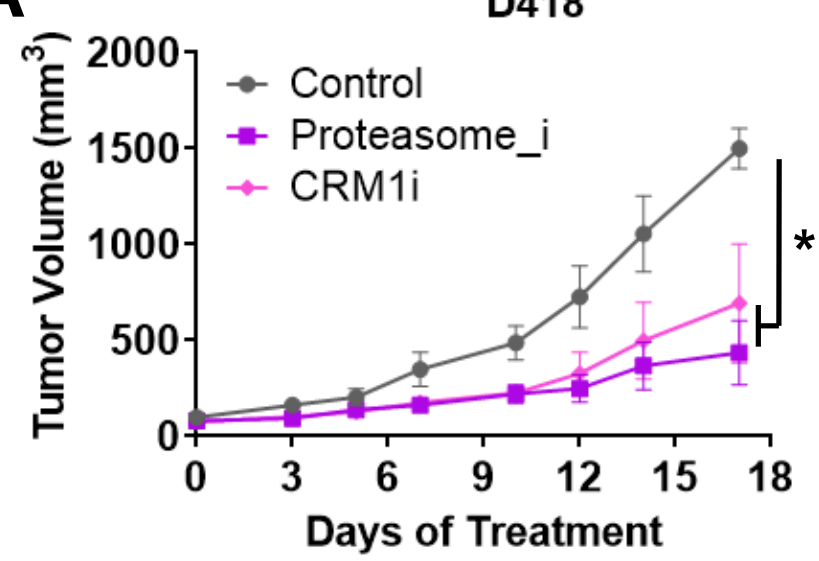

C

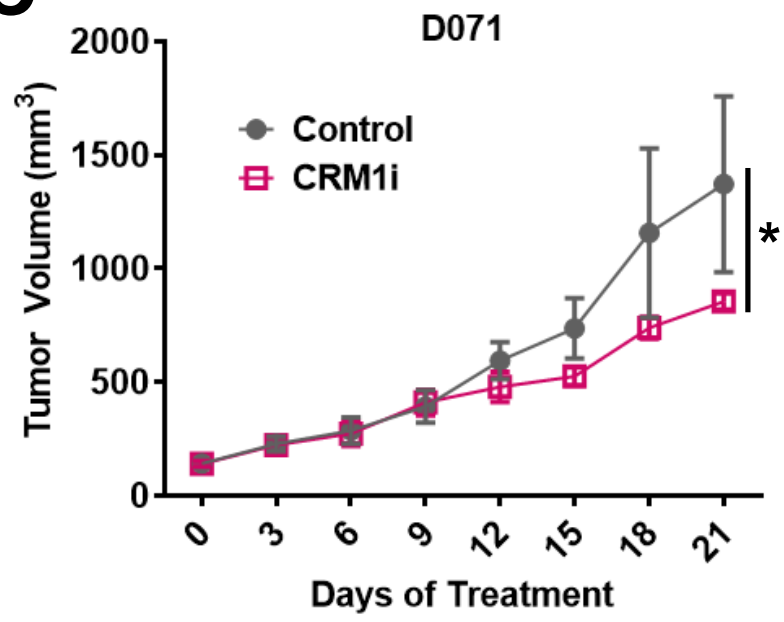

E

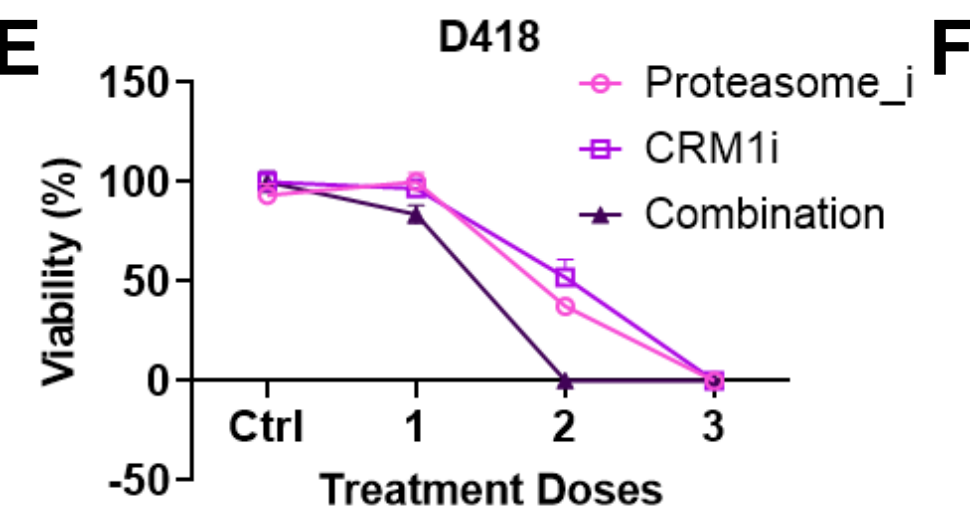

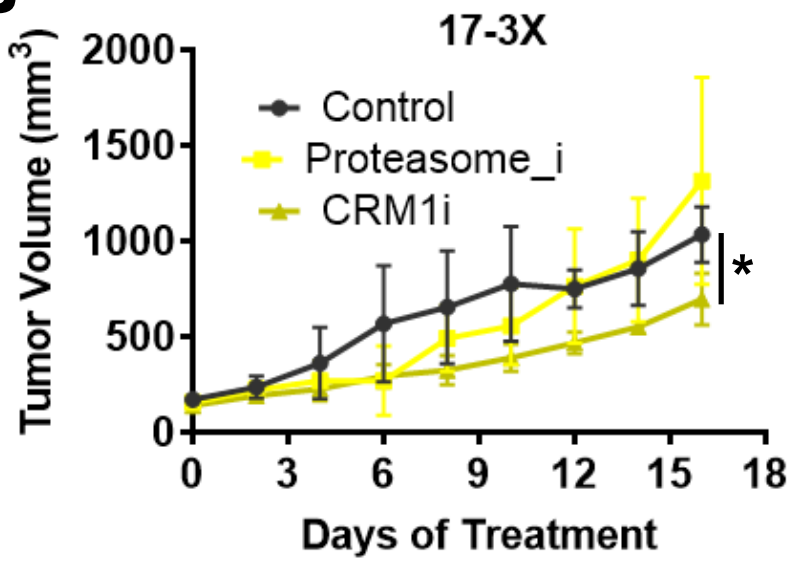

D

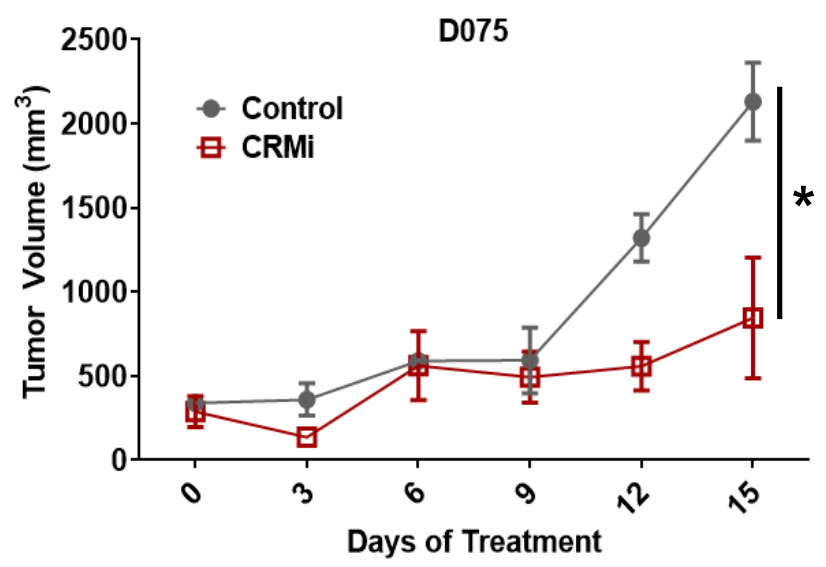

17-3X

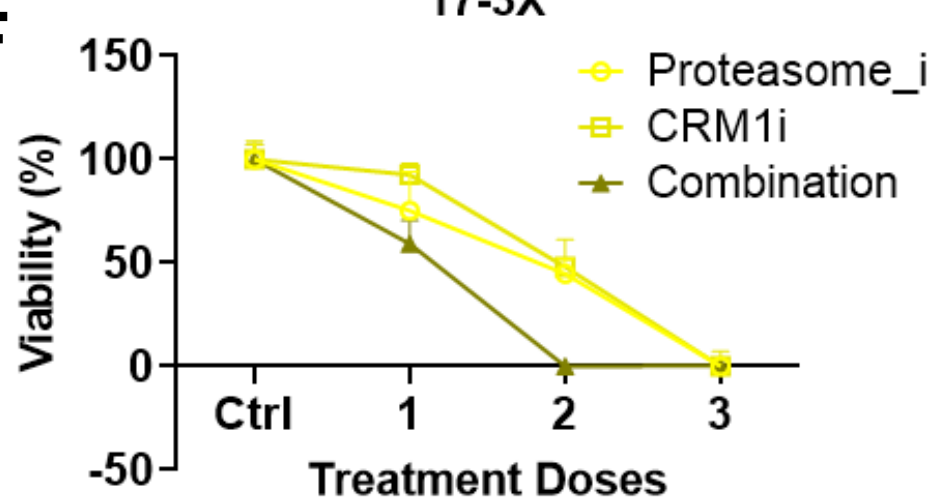


B Human

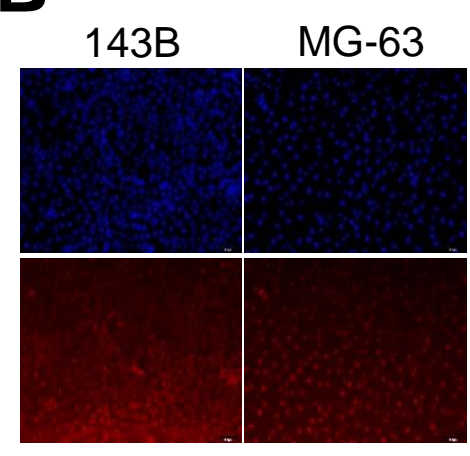

D

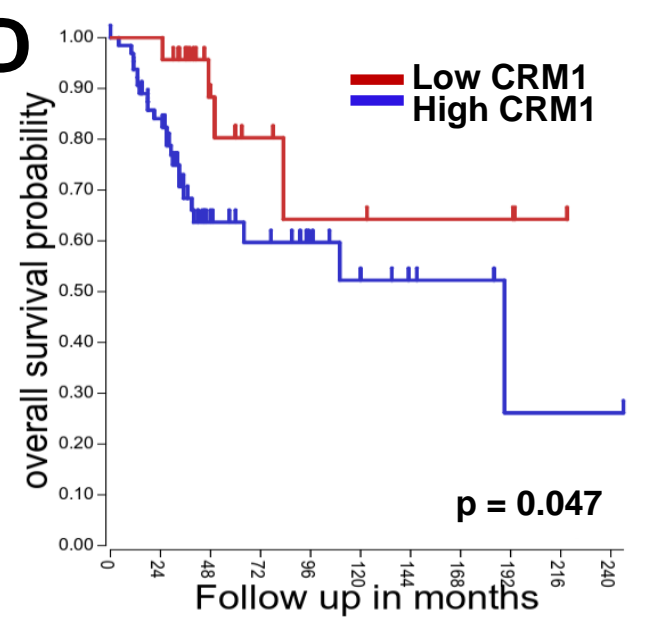

F

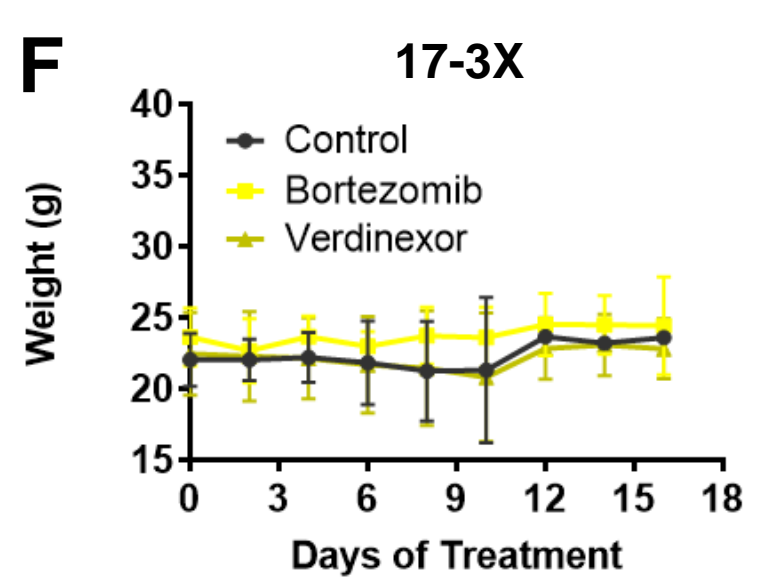

E

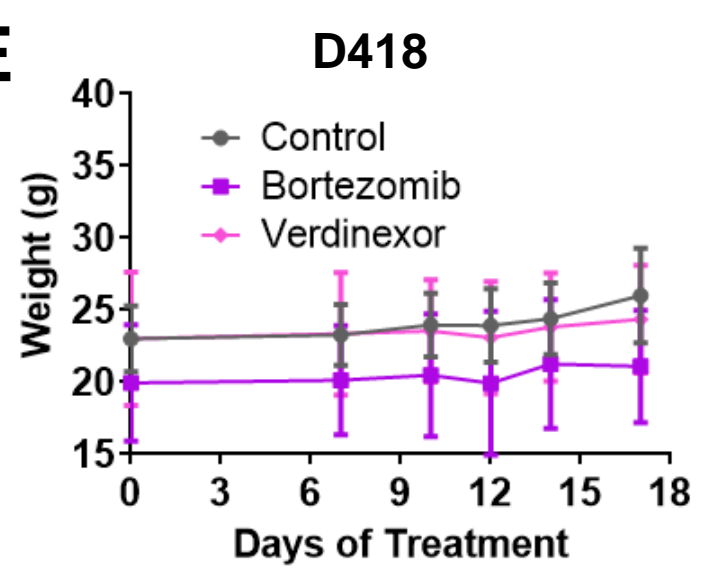

Dog
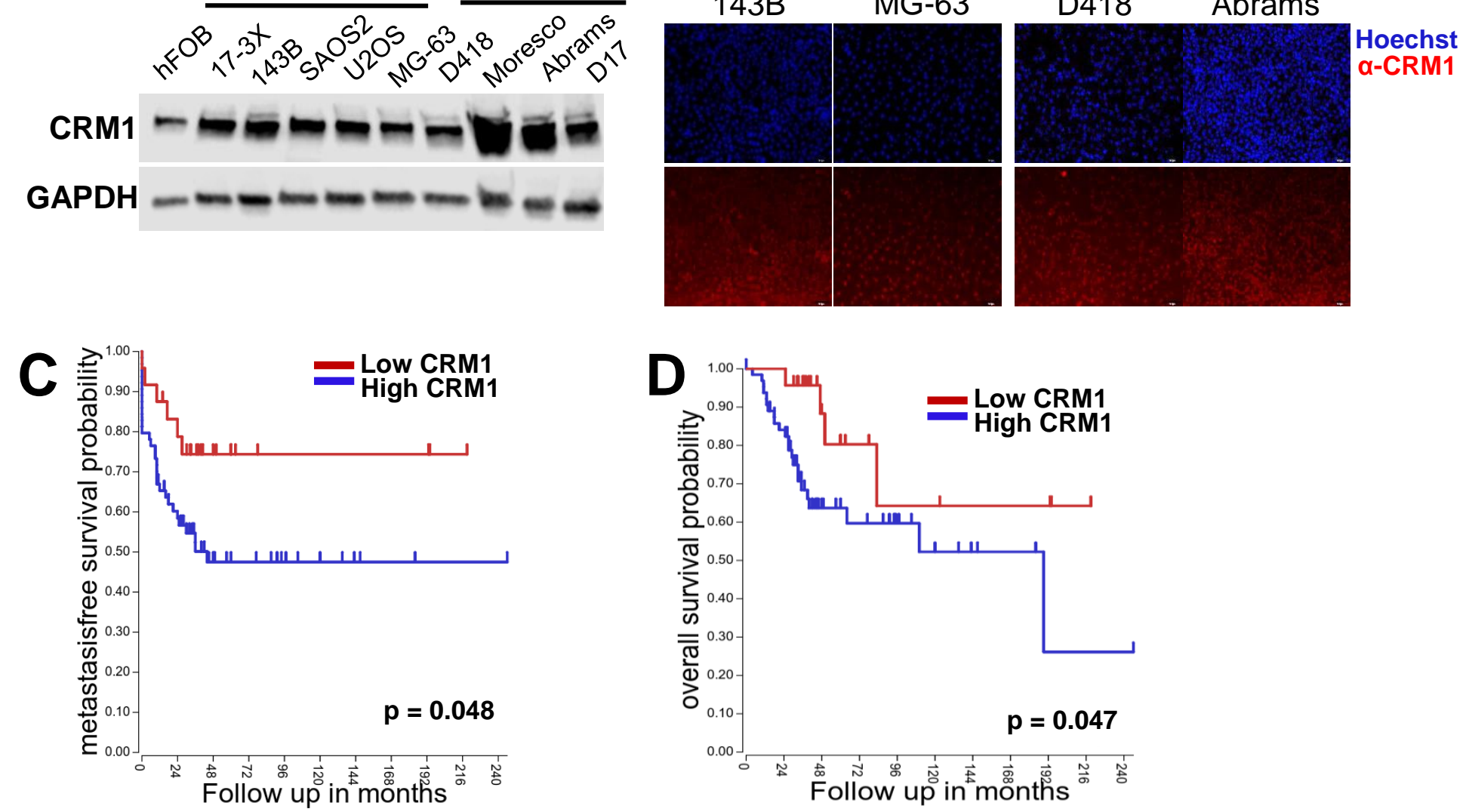